Diabetologe 2021 · 17:638-646

https://doi.org/10.1007/s11428-021-00758-4

Angenommen: 1. April 2021

Online publiziert: 22. April 2021

(C) Springer Medizin Verlag GmbH, ein Teil von Springer Nature 2021

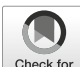

Check for
updates

\author{
Simone von Sengbusch ${ }^{1} \cdot$ Fabian S. Frielitz $^{2} \cdot$ Katarina Braune $^{3} \cdot$ Karina Boss $^{3} \cdot$ \\ Klemens Raile ${ }^{3}$ \\ ' Klinik für Kinder- und Jugendmedizin, Pädiatrische Endokrinologie und -diabetologie, \\ Universitätsklinikum Schleswig-Holstein, Campus Lübeck, Lübeck, Deutschland \\ ${ }^{2}$ Institut für Sozialmedizin und Epidemiologie, Universität zu Lübeck, Lübeck, Deutschland \\ ${ }^{3}$ Klinik für Pädiatrie m. S. Endokrinologie und Diabetologie, Campus Virchow Klinikum, Charité - \\ Universitätsmedizin Berlin, Berlin, Deutschland
}

\title{
Telemedizin in der Kinderdiabetologie
}

\section{Videosprechstunden als Erweiterung der ambulanten Diabetesversorgung}

lichen mit Diabetes sowie ihre Familien, entscheidend zum Erfolg des nächsten Innovationsschritts in der Kinder- und Jugenddiabetologie bei. Mit diesem Artikel geben wir einen Überblick über bereits bestehende Projekte und skizzieren daskonkrete Entwicklungspotenzial telemedizinischer Innovationen in Deutschland.

\section{Implementierung der Videosprechstunde in die pädiatrische Diabetesambulanz}

In den letzten 2 Jahrzehnten leisteten die Kinderdiabetolog:innen und Diabetesberater:innen Pionierarbeit für ihre jungen Patient:innen, indem sie früh sinnvolle, neue Medizintechnologien in die Versorgung von Kindern und Jugendlichen mit Diabetes integrierten. Die Nutzung von Sensoren zur kontinuierlichen Glukosemessung (CGM [,,continuous glucose measurement"]) und Insulinpumpen nahm schnell und kontinuierlich zu, wobei die jüngsten Kinder die meiste Anwendung von Medizintechnik erfahren [4]. Diese Entwicklung der Closed-Loop-Insulinpumpensysteme im Blick scheint der Zeitpunkt gekommen, den vernetzten Einsatz dieser Techniken in die kontinuierliche Versorgung $\mathrm{zu}$ realisieren. Zunehmend mehr Diabetesteams stehen einer Einführung von Videosprechstunden in die Regelversorgung positiv gegenüber, auch wenn deren Verbreitung noch nicht sehr hoch ist [5].

\) Immer mehr Diabetesteams
befürworten die Einführung
von Videosprechstunden in die
Regelversorgung

Die Etablierung einer Videosprechstunde als ergänzendes Angebot erfordert v. a. in Krankenhäusern längere Implementierungsprozesse, deren Geschwindigkeit an

I. Prüfung, Einkauf und Etablierung datenschutzkonformer Videoportale und Datenplattformen,

II. neu ausgerichtete Arbeitsabläufe und Prozesse,

III. digitale Kompetenz - insbesondere IT-Kompetenz (IT: Informationstechnologie) und Fähigkeiten im Bereich digitales Lernen und Lehre - des medizinischen Personals und letztendlich

IV. ökonomische Entscheidungen der Klinikbetreiber:innen

gebunden ist.

Im Einzelnen stellen, nach einem erfolgreichen Start, die Einbindung neuer Prozesse und deren Organisation eine erhebliche Herausforderung dar. Die Einführung von Softwarelösungen zu den verschiedenen CGM-Systemen und In- 
Hier steht eine Anzeige.

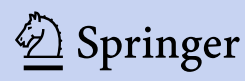


sulinpumpen ist ein Prozess, der ein abgestimmtes Verfahren innerhalb einer Klinik mit der IT (Verfahrensbeschreibung), den Datenschutzbeauftragten und den Jurist:innen der Klinik erfordert. Ein Beispiel eines Datenschutzkonzepts wurde im Rahmen der ViDiKi-Studie (ViDiKi: „Virtuelle Diabetesambulanz für Kinder und Jugendliche") publiziert und dient zur Orientierung für zukünftige Projekte [6].

Die Einführung der Videosprechstunde verändert auch die Arbeitsorganisation des Diabetesteams in der klinischen Praxis. Neben der Einrichtung geeigneter Arbeitsplätze mit der entsprechenden technischen Ausstattung und in ruhiger Umgebung stellen sich auch die Frage der interdisziplinären Betreuung mit Delegation des Beratungsanteils an Diabetesberater:innen sowie die generelle Möglichkeit, auf Wunsch einen Teil der Beratungszeit im Homeoffice leisten zu können.

Für die Familien mit einem an Typ-1Diabetes erkrankten Kind bietet die ergänzende Videosprechstunde zum persönlichen Kontakt die Möglichkeit häufigerer Kontakte mit Zeitersparnis durch die fehlende Anfahrt und Durchlaufen bürokratischer Prozesse in Klinik oder Praxis. Für Diabetesteams gilt es nun, gemeinsam Konzepte zu entwickeln, wie sie diese neuen Technologien, die dafür nötigen Softwarelösungen und Kommunikationsmedien in ihr bisheriges, individuelles Versorgungskonzept integrieren.

Eine offene Haltung der Akteur:innen in der medizinischen Versorgung wird sich als positiv erweisen, da sich der Markt der telemedizinischen Betreuungsleistungen rasch entwickelt und schon jetzt kommerzielle Anbieter:innen Diabetes-Coaching und -schulungen durchführen. Auch der Markt der digitalen Gesundheitsanwendungen (DiGA) wird die Angebote der Versorgungsleistungen erweitern.

\section{Rechtliche Rahmen- bedingungen}

Durch die Verabschiedung des neu gefassten $₫ 7$ Absatz 4 (Muster-)Berufsordnung für Ärzt:innen (MBO-Ä) durch den Ärztetag im Mai 2018 wurden weitestge- hend die Hürden für die telemedizinische Versorgung gelöst, indem das Fernbehandlungsverbot aufgehoben wurde. Somit ist eine

ausschließliche Beratung oder Behandlung über Kommunikationsmedien ... im Einzelfall erlaubt, wenn dies ärztlich vertretbar ist und die erforderliche ärztliche Sorgfalt insbesondere durch die Art und Weise der Befunderhebung, Beratung, Behandlung sowie Dokumentation gewahrt wird und die Patientin oder der Patient auch über die Besonderheiten der ausschließlichen Beratung und Behandlung über Kommunikationsmedien aufgeklärt wird. Gemäß $₫ 7$ Abs. 4 Satz 3 MBO-Ä. [7]

Gleichzeitig wurden durch die kassenärztliche Bundesvereinigung gewisse Anforderungen an die telemedizinisch gestützte Betreuung von Patient:innen formuliert [8]:

1. Der zu Behandelnde muss für die Videosprechstunde eine Einwilligung abgeben.

2. Die Videosprechstunde muss in Räumlichkeiten stattfinden, die eine Privatsphäre gewähren. Zudem muss die eingesetzte Technik eine angemessene Kommunikation erlauben.

3. Die Videosprechstunde muss vertraulich und störungsfrei verlaufen, d.h. in Art und Weise der normalen Sprechstunde.

4. Der Klarname des Patient:in muss für den Behandelnden erkennbar sein.

5. Die Videosprechstunde muss frei von Werbung sein.

6. Es muss ein zertifizierter Videodienst, welcher eine verschlüsselte End-zuEnd-Übertragung gewährleistet, eingesetzt werden.

Die technischen Anforderungen für die Praxis und den Videodienstanbieter:in im Hinblick auf die technische Sicherheit und den Datenschutz wurden in der Anlage 31b zum Bundesmantelvertrag - Ärzte geregelt. Ärzt:innen oder Psychotherapeut:innen können Leistungen nur dann honoriert bekommen, wenn sie ihrer kassenärztlichen Vereinigung zuvor angezeigt haben, einen zertifizierten Videodienstanbieter:in zu nutzen. Dabei sollte beachtet werden, dass mit Wirkung zum 20.03.2021 eine Überarbeitung der Anlage in Kraft tritt, diese betrifft weitestgehend die Anforderungen zur ITSicherheit und zum Datenschutz der Zertifizierung des Anbieter:in. Bereits zertifizierte Anbieter:innen könnten weiter eingesetzt werden und müssen im Rahmen einer Übergangsfrist die neuen Anforderungen erfüllen.

\section{》) Die rechtlichen Fragen bei cloudbasierten Anwendungen sollten juristisch geprüft werden}

Cloud-Computing ist dadurch gekennzeichnet, dass Informationstechnologien nicht mehr lokal von den Nutzer:innen selbst vorgehalten werden. Sie werden stattdessen dezentral über Netzwerkstrukturen bereitgestellt. Das Gesundheitswesen hat im Vergleich zu anderen Branchen noch Nachholbedarf in der Nutzung und Umsetzung neuer Technologien. Dies ist auf einen stark regulierten Markt zurückzuführen.

$\mathrm{Zu}$ den cloudbasierten Informationstechnologien gehören: Speicherplatz und Rechenleistung, aber auch Anwendungsprogramme bis hin zur Nutzung künstlicher Intelligenz. Die Diabetologie ist durch eine stark wachsende Digitalisierung geprägt. Der Wunsch der Anwender:innen, ihre Daten in Echtzeit auf den verschiedenen Geräten, wie Smartphone oder Smartwatch, mitlesen zu können, ist groß. Ebenso ist die cloudbasierte Speicherung der aufgezeichneten CGM-Daten neben der lokalen Speicherung gefragt. Auch hier stellen sich rechtliche Fragen, z. B. wo sich eingesetzte Server, auf dem die CGM-Daten gespeichert werden, befinden oder wann ein AV-Vertrag (AV: Auftragsverarbeitung) notwendig ist [9].

Cloudmedizin stellt im Rahmen der telemedizinischen Beratung einen Baustein dar, um Patient:innen mittels Videoportal beraten zu können. Denn nur, wenn aktuelle Therapiedaten zur Verfügung stehen, kann eine Therapieempfehlung durch Ärzt:innen im Rahmen der Fernbehandlung erfolgen. Die Anforderungen bezüglich AV-Verträgen, Technikeinsatz und Datenschutz sollten juristisch geprüft werden. 


\section{Praktische Beispiele und ihre Entwicklungsgeschichte}

\section{ViDiKi in Schleswig-Holstein}

Dem Innovationsfondsprojekt „Virtuelle Diabetesambulanz für Kinder und Jugendliche", ViDiKi (2017-2020), ging eine Pilotstudie, Duo-SuP, voraus. An dieser hatten, im Zeitraum 2013-2014, 5 Familien mit jeweils 2 an Typ-1Diabetes erkrankten Geschwistern die damals noch neuartige Versorgung mit einer sensorunterstützten Pumpentherapie der 1. Generation erprobt und 2-wöchentlich telefonisch eine Beratung zu CGM- und Insulinpumpendaten erhalten [10]. Die Weiterentwicklung der Hilfsmittel und cloudbasierte Softwarelösungen, das Vorbild der Diabeteskliniken in den Niederlanden [11] und v. a. die Entscheidung des G-BA (Gemeinsamer Bundesausschuss) im September 2016, die rtCGM-Technologie (rtCGM: „realtime continuous glucose measurement") in die Regelversorgung $\mathrm{zu}$ überführen, ermutigten zu einem Antrag beim Innovationsfonds zur Erprobung der Videosprechstunde auf Basis von CGMAuswertungen. Die in der ersten Innovationsfondswelle geförderte ViDiKiStudie war eine quasi randomisierte, multizentrische, prospektive Studie unter realen Versorgungsbedingungen in Schleswig-Holstein und Hamburg. Es wurden 240 Kinder und Jugendliche (1-16 Jahre) mit Typ-1-Diabetes eingeschlossen, die alle bei Studienstart bereits ein CGM- oder rtCGM-System nutzten. In der 6-monatigen kontrollierten ersten Studienphase erhielten nur die Kinder der Interventionsgruppe das Angebot der monatlichen Videosprechstunde zusätzlich zur Regelversorgung in der Diabetesambulanz (• Abb. 1). In dieser Phase kam es nur zu einer geringen Verbesserung des $\mathrm{HbA}_{1 \mathrm{c}}$ (glykiertes Hämoglobin Typ $A_{1 c}$ ) in der Interventionsgruppe, aber zu einer signifikanten Verbesserung der Therapiezufriedenheit der Eltern und Reduktion der mütterlichen Belastung [12]. Betrachtete man die gesamte Kohorte aller Kinder, von denen knapp 1/3 schon bei Studieneintritt eine gute Stoffwechsellage aufwies, konnte nach 1 Jahr eine signifikante und

Diabetologe 2021 · 17:638-646 https://doi.org/10.1007/s11428-021-00758-4

๑) Springer Medizin Verlag GmbH, ein Teil von Springer Nature 2021

\section{S. von Sengbusch · F. S. Frielitz · K. Braune · K. Boss · K. Raile}

\section{Telemedizin in der Kinderdiabetologie. Videosprechstunden als Erweiterung der ambulanten Diabetesversorgung}

\section{Zusammenfassung}

Der aktuelle Stand telemedizinischer Anwendungen und deren zu erwartendes Potenzial werden aus der Perspektive der pädiatrischen Diabetologie beleuchtet. Kontinuierliche Glukosemesssysteme (CGM ["continuous glucose measurement"]) und Insulinpumpen lassen sich zunehmend auch über interoperable oder herstellerbasierte Datenplattformen auslesen und teilen und ermöglichen es den Diabetesteams, eine Beratung auf Basis dieser Daten auch ohne physischen Kontakt in die kontinuierliche Versorgung einzubauen. Videosprechstunden können dabei zunächst ein ergänzendes Angebot darstellen, unterliegen oft längeren Implementierungsprozessen und sind u. a. abhängig von datenschutzkonformer
Videochatsoftware, koordinierten Arbeitsabläufen sowie technischem Support. Telemedizin kann derzeit schon bestehende Beratungsstrukturen in Kliniken und Praxen ergänzen und helfen, das Potenzial digital dokumentierter Daten aus CGM- und automatisierten Insulinabgabesystemen voll auszuschöpfen, wobei telemedizinische Anwendungen bisher kein direktes Einsparungspotenzial bezüglich Zeit und Aufwand des medizinischen Personals und damit der Personalkosten bieten.

Schlüsselwörter

E-Health · Typ-1-Diabetes · Kind · Jugendlicher - Digitale Technologie

\section{Telemedicine to enhance pediatric diabetes care. Video consultations as an extension of outpatient diabetes care}

\section{Abstract}

We present the current status and potential of currently available telemedicine concepts with a specific focus on pediatric diabetes care. Data from continuous glucose measurement (CGM) systems and insulin pumps can increasingly be downloaded and shared via interoperable or manufacturer-based data platforms, and thus enable health care professionals of diabetes teams to provide advice based on these data for continuous medical care, even if not met in-person. Initially, video consultations may represent an additional service, as they are often subject to long implementation processes and depend, among other things, on video chat software that is compliant to data privacy and security, coordinated operating processes and finally, technical support. Telemedicine can currently support existing consultation concepts in hospitals and outpatient clinics and thus help fully utilize the potential of digital documentation of CGM and automated insulin devices. However, it is important to note that telemedicine has not yet proven a reduction in time and effort for medical staff and impact on personnel costs.

\section{Keywords}

eHealth · Type 1 diabetes · Child · Adolescent . Digital technology klinisch relevante Reduktion des $\mathrm{HbA}_{1 \mathrm{c}}$ erreicht werden [12].

\section{"Digital Diabetes Clinic" in Berlin}

In diesem Pilotprojekt [13] wurden an der Charité - Universitätsmedizin Berlin 28 Kinder mit Typ-1-Diabetes über einen Zeitraum von 6 Monaten hinweg „virtuell“ betreut, davon jeweils 3 Monate vor und während des ersten Lockdowns der COVID-19-Pandemie (COVID-19: „coronavirus disease 2019“). Das Stu- diendesign wurde gemeinsam mit allen Therapeut:innen des Diabetesteams, unseren Patient:innen und deren Familien mit Unterstützung durch ein Team aus Servicedesigner:innen entwickelt [13]. Alle Teilnehmer:innen schlossen die Studie erfolgreich ab und konnten ihre Zeit der Sensorglukosewerte im Zielbereich („time in range“) signifikant um $+11 \%$ verbessern und Unterzuckerungen reduzieren. Weiterhin konnten wir trotz des Lockdowns eine Verbesserung der diabetesspezifischen und psychosozialen 


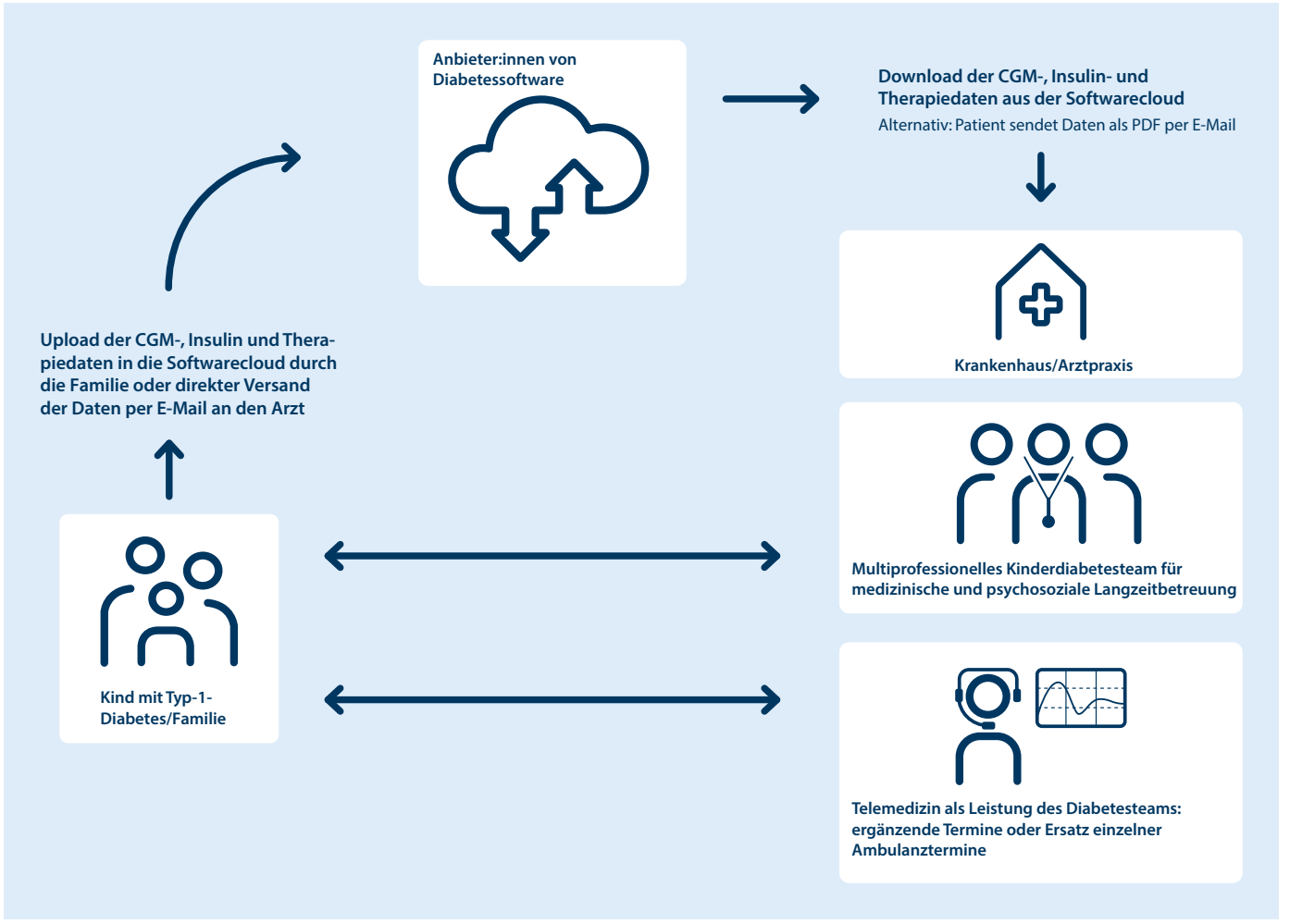

Abb. 1 - Ergänzung der Regelversorgung durch Videosprechstunde auf Basis online verfügbarer Diabetestherapiedaten, CGM kontinuierliche Glukosemessung, $P D F$,portable document format". (Design und Entwurf Grafikabteilung, UKSH [Universitätsklinikum Schleswig-Holstein])

Gesundheit feststellen. Die Eltern eines Studienteilnehmers formulierten es wie folgt:

Durch die monatlichen Termine haben wir regelmäßiger die Therapieeinstellungen überprüft und ggf. angepasst. Uns wurde häufiger etwas Entscheidungsdruck abgenommen, da wir fundiertes Feedback bekamen. Der fixe Termin einmal im Monat ohne Anreise hilft enorm [14].

\section{Zukunftschancen der Telemedizin}

\section{Einzel- und Gruppenschulungen per Video}

In der Coronapandemie ließen Diabetesteams Schulungsleistungen, die einen erheblichen Anteil des Versorgungsspektrums ausmachen, entweder ausfallen oder boten diese, sofern möglich, per Telefon oder online an, da die Schulung in Präsenz für diese Zwecke nur im reduzierten Rahmen möglich war.

Eine große Herausforderung stellen hier die Schulungen in Gruppen von Kindern und Jugendlichen dar. Kinder und Jugendliche entwickeln ab dem
12. Lebensjahr ausreichend Medienkompetenz, um aktiv und sicher an Onlineformaten teilzunehmen. Insbesondere wenn schon Erfahrungen mit E-Learning bestehen, stellen virtuelle Schulungs- und Empowermenteinheiten (Empowerment: Förderung der Fähigkeit für selbständiges/selbstbestimmtes Handeln) ein bisher ungenutztes Potenzial dar und entsprechen dem etablierten Medienverhalten dieser Altersgruppe [15].

\section{》) Kinder können ab etwa dem \\ 12. Lebensjahr aktiv und sicher an Onlineformaten teilnehmen}

Die bisher meist erlebnisorientiert gestalteten Gruppenschulungen in Klinik und Praxis können und sollen allerdings perspektivisch nicht vollständig durch Onlinegruppenschulungen ersetzt werden, denn die Schulungseffekte inklusive einer positiven Gruppendynamik, Zugehörigkeitsgefühl und Peer-to-Peer-Effekten sind $\mathrm{zu}$ wertvoll $[12,14,16]$.

Erfolgreiche Onlinegruppenschulungen in der pädiatrischen Diabetologie erfordern außerdem eine angepasste Fach- didaktik sowohl im curricularen-inhaltlichen als auch im didaktisch-methodischen Bereich und eine erweiterte $\mathrm{Me}$ dienkompetenz aller Akteur:innnen. Bei diesen Weiterentwicklungen sollten jedoch die grundlegenden Empfehlungen, wie z.B. Kompetenzorientierung, Binnendifferenzierung und Empowermentansatz, weiterhin im Fokus behalten werden.

\section{Beratung für sozialmedizinische Fragestellungen}

Diabetesfachorganisationen und $\mathrm{Pa}$ tient:innenorganisationen nutzen schon seit Jahren die verfügbaren Medien, um Patient:innen im komplexen Gebiet der psychosozialen und sozialrechtlichen Fragestellungen rund um Diabetes mit Rat zur Seite zu stehen. Am häufigsten werden dabei verschriftlichte Informationen, aber auch Informationsaustausch per Chat, Social Media und Telefon angeboten. Digitale Technologien bieten auch hier nicht nur erweiterte Kommunikationsmöglichkeiten, sondern auch wohnortunabhängigen Zugang zu spezialisierter Beratung. Im Rahmen der von der Damp-Stiftung geförderten 
Hier steht eine Anzeige.

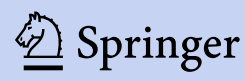


Internetpräsenz und Funktion der Kinderdiabeteslots:innen für SchleswigHolstein [17] wurden für dieses Bundesland Versorgungspfade analysiert und eine Informationsplattform geschaffen, die Expert:innen und Patient:innen gleichermaßen Information und Kontakt bietet und inzwischen auch überregional Aufmerksamkeit erweckt.

\section{Weitere gemeinsame Optimierung durch alle Akteur:innen}

In den letzten 20 Jahren kam es durch neue Medizinprodukte und Insuline $\mathrm{zu}$ erheblichen Weiterentwicklungen der therapeutischen Möglichkeiten bei Typ1-Diabetes im Kindes- und Jugendalter. Zugleich wurden die Schulungen bei Manifestation und Therapieumstellung komplexer. Spezielle Hilfsmittel wie z. B. CGM-Geräte, sensorgesteuerte oder (teil-)automatisierte Insulinpumpen machen eine umfassende Schulung der Patient:innen und ihrer Familien zum Therapiebeginn erforderlich. Der Einsatz dieser Hilfsmittel ermöglicht ein von Anfang an besseres Verständnis für dynamische Effekte von Insulin, Ernährung und Bewegung auf die unmittelbaren sich verändernden Glukosespiegel. Aus den CGM-Daten konnten Patient:innen und Diabetesteams aber auch erstmalig die tatsächliche Realität der Glukosedynamik ablesen. Eine Insulinanpassung nur alle 3 Monate in der Spezialambulanz wird, erkennbar an der Entwicklung der Glukosewerte in den Tag-für-Tag- und Trendprofilen, dem variablen Insulinbedarf eines wachsenden Kindes nicht gerecht. Häufigere Kontakte zur dynamischen Insulinanpassung sind in vielen Abschnitten der Langzeitbetreuung sinnvoll, aber aufgrund von Personalmangel oft nur bedingt umsetzbar.

\section{) Mittels Telemedizin kann das Selbstmanagement von Menschen mit Diabetes optimal gefördert werden}

Wir sehen in der Telemedizin eine ideale Komponente, das Selbstmanagement von Menschen mit Diabetes optimal zu fördern. Die Telemedizin kann perspektivisch durch das Angebot von Telefon, E-Mail und v.a. Videokontakten bedarfsgerecht häufigere, etwas kürzere, auf das Wesentliche konzentrierte Beratungstermine ermöglichen, ohne dass Patient:innen dafür in die Klinik oder Praxis fahren müssen. Die Zeitersparnis auf Seiten des Diabetesteams fällt zwar aktuell noch gering aus, allerdings könnte sich hier die zu erwartende Softwareweiterentwicklung mit automatischer Analyse und Therapievorschlägen für die Ärzt:innen zeitersparend auswirken.

Die Videosprechstunde als Ersatz für eine Folge von Quartalsambulanzkontakten hat aber auch Grenzen. Bei einigen Patient:innen weicht der aus CGMWerten errechnete Glukosemanagementindikator (GMI) erheblich vom Labor$\mathrm{HbA}_{1 \mathrm{c}}$-Wert der Klinik bzw. Praxis ab, sodass eine Beratung allein auf Basis der CGM-Profile nur bedingt möglich ist. Zuhause durchführbare $\mathrm{HbA}_{1 \mathrm{c}}$-Tests können eine Hilfe für die Patient:innen darstellen, bei denen der Labor-HbA $\mathrm{lc}^{-}$ Wert erheblich vom GMI differiert, allerdings müssen die Betroffenen in deren Anwendung gut geschult werden, um präanalytische Fehler zu vermeiden. Weiterhin entfallen bei der Videosprechstunde Kontrollen von Blutdruck, Länge und Gewicht, und eine rein visuelle Kontrolle der Spritz-/Katheterstellen ist nur bedingt möglich, da sich Verhärtungen im Gewebe optisch nicht abzeichnen.

Häufig wird die Sorge thematisiert, dass Analysesoftware für CGM-Daten und Closed-Loop-Systeme das Diabetesteam in naher Zukunft überflüssig machen könnten, das Berufsbild sich sozusagen durch Digitalisierung der Therapiedaten selbst „wegrationalisiert“. Richtig ist, dass sich die Betreuung mit der zunehmenden Verbreitung von Technologien weiter verändern wird. Die persönliche und individuelle Betreuung durch Diabetesteams wird jedoch keineswegs überflüssig werden. Neue Betreuungsmodelle, die flexible Modelle mit Präsenz- und Videokontakten (- Abb. 1) zulassen, erwiesen sich z.B. in der ViDiKi- sowie in der DDC-Studie als sehr positiv und effektiv und ermöglichen eine höherfrequente, flexiblere, an die individuellen Therapieformen, die Patient:innen gegenwärtig und zukünftig zur Verfügung stehen, angepasste Betreuung. Die Schulung, die Langzeitbetreuung, die psychosoziale Beratung sowie die Behandlung von weiterhin möglichen diabetesassoziierten Folgeund Begleiterkrankungen werden dabei die Kernkompetenz der Diabetesteams bleiben. Die Herausforderung, die Art der Langzeitbetreuung von Menschen mit Diabetes neu zu denken und $\mathrm{zu}$ gestalten, kommt zeitgerecht zum Innovationsschub in der Diabetesbehandlung. Aktuell kann zwar noch nicht von einer Zeitersparnis auf Seiten des Diabetesteams durch die Anwendung von Telemedizin ausgegangen werden, zukünftige technische Entwicklungen im Bereich Datenanalyse und Therapieanpassung aber haben das Potenzial, zeitliche Einsparungen zu erwirken.

In unterversorgten Regionen könnte die kooperierende Versorgung durch ein entferntes Kinderdiabeteszentrum unter Nutzung von Telemedizin eine Chance für die Familien darstellen, die sonst keinen Zugang zu einem multiprofessionellen Team hätten. Die Zusammenarbeit sollte allerdings formal und inhaltlich abgestimmt sein, wenn mehrere Therapeut:innen für Kinder mit Diabetes einen Teil der Versorgung übernehmen. Die Trennung in finanziell attraktive technikorientierte Einzelleistungen durch kommerzielle Anbieter:innen und der Verbleib der schon jetzt unterfinanzierten, personalintensiven, medizinischen und psychosozialen Beratungsaufgaben des Diabetesteams müssen aktiv adressiert und bei der Ausgestaltung von Versorgungsverträgen für Kinder und Jugendliche mit Diabetes entsprechend berücksichtigt werden. Zudem bietet die Telemedizin die Chance, dem „Nachwuchsproblem" und dem zukünftig absehbaren Mangel an Fachärzt:innen im Bereich der diabetologischen Versorgung sowohl für Kinder und Jugendliche als auch für Erwachsene vorausschauend zu begegnen. 
Erfordernis einer adäquaten

Vergütung der hochspezialisierten Telemedizin für Kinder

Familien sind mit dem Diabetestherapiemanagement ihrer Kinder oft überfordert und benötigen intensive therapeutische und psychosoziale Unterstützung. Der steigende Bedarf an psychologischer Betreuung und sozialen Hilfen gerade nach Manifestation der Erkrankung ist bisher gesundheitsökonomisch nicht entsprechend eingepreist. Ursachen für die Verschärfung des Leistungsdrucks sind mehr sehr junge Kinder mit Diabetes, sich verändernde Familienstrukturen, geringere Deutschkenntnisse und/ oder niedriges Bildungsniveau bzw. niedriger sozioökonomischer Status der Sorgeberechtigten. Hinzu kommt die zunehmend verschärfte Finanzlage, insbesondere pädiatrischer Abteilungen, und dieser Kostendruck führt in erster Linie zu Einsparungen in nichtärztlichen Berufsgruppen.

Vor diesem Hintergrund ist sicherzustellen, dass die Versorgungsstrukturen in der Kinder- und Jugenddiabetologie neu und adäquat von Kostenträger:innen und Leistungserbringer:innen unterstützt werden. Die Telemedizin und hier die Videosprechstunde als neue Beratungsform müssen als Extraleistung ausreichend finanziert sein, sonst werden sie sich, trotz positiver Studienlage, in der Regelversorgung nicht etablieren können.

\section{Fazit für die Praxis}

- Die Speicherung, das Zusammen-

führen und das grafische Aufbereiten von Therapiedaten durch Software ermöglichen Telemedizin in einem Rahmen, der einer ambulanten Gesprächssituation sehr nahekommt und in einer breiten $\mathrm{Pa}$ tient:innengruppe eingesetzt werden kann.

- Die Videosprechstunde war schon vor der Coronapandemie ein erfolgreiches Kommunikationsmedium, um Extrakontakte zur Therapieanpassung zu ermöglichen.
- Die Zeiteinsparung und die höherfrequente Beratung mit Insulinanpassung sind die größten Vorteile für Patient:innen, v. a. in Lebenssituationen, die einen intensiven und häufigen Austausch nötig machen.

- Telemedizin sollte bestehende Beratungsstrukturen in Kliniken und Praxen zunächst ergänzen, damit das Potenzial der CGM-Therapiebegleitung (CGM: kontinuierliche Glukosemessung) und zunehmend automatisierten Insulintherapie voll ausgeschöpft wird.

- Bisher zeichnet sich durch telemedizinische Anwendungen kein Einsparungspotenzial für medizinisches Personal ab.

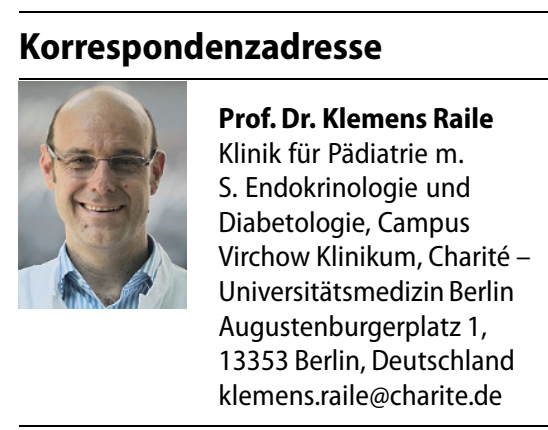

\section{Einhaltung ethischer Richtlinien}

Interessenkonflikt. S. von Sengbusch ist im „Advisory Board" für die Firmen Abbott, Lilly, Medtronic und Novo Nordisk tätig. Sie erhielt in den letzten 2 Jahren Vortragshonorare und Reisekostenerstattung durch die Firmen Abbott, Berlin-Chemie, Dexcom, Hexal, Infectopharm, Lilly, Medtronic, Novo Nordisk und Sanofi. Sie leitete die ViDiKi-Telemedizinstudien und wird durch die Damp-Stiftung im Rahmen des Kinderdiabeteslots:innenprojektes gefördert. K. Braune und K. Raile erhielten Forschungsförderung durch das „European Commission's Horizon 2020 Research and Innovation Programm". K. Braune erhielt Förderung durch das DFG-geförderte (DFG: Deutsche Forschungsgemeinschaft) „Berlin Institute of Health (BIH) Digital Clinician Scientist Programm “, ,BIH Junior Clinician Scientist Programm" sowie durch den „SPOKES Wellcome Trust", Stiftung Charité und Deutsche Diabetes Gesellschaft (DDG); ohne Zusammenhang mit dem Artikel. Sie erhielt weiterhin innerhalb der letzten 3 Jahre Honorare für Consulting und Talks von Roche Diabetes Care, Dexcom, Medtronic Diabetes und Diabeloop. K. Raile ist "Advisory Board Member" bei Lilly Diabetes Care und Abbott Diabetes Care, er erhielt Vortragshonorare von Abbott Diabetes Care, Novo Nordisk, Sanofi und Springer Healthcare IME. F.S. Frielitz und K. Boss geben an, dass kein Interessenkonflikt besteht.

Für diesen Beitrag wurden von den Autoren keine Studien an Menschen oder Tieren durchgeführt.
Für die aufgeführten Studien gelten die jeweils dort angegebenen ethischen Richtlinien.

\section{Literatur}

1. Neu A, Burger-Busing J, Danne T, Dost A, Holder M, Holl RW, Holterhus PM, Kapellen T, Karges B, Kordonouri $O$ et al (2019) Diagnosis, Therapy and Follow-Up of Diabetes Mellitus in Children and Adolescents. Exp Clin Endocrinol Diabetes 127:S39-S72

2. Doger E, Bozbulut R, Soysal AAS, Ercan S, Kilinc Ugurlu A, Akbas ED, Bideci A, Camurdan O, Cinaz P (2019) Effect of Telehealth System on Glycemic Control in Children and Adolescents with Type 1 Diabetes. JClin Res Pediatr Endocrinol 11:70-75

3. Crossen S, Glaser N, Sauers-Ford H, Chen S, Tran V, Marcin J (2020) Home-based video visits for pediatric patients with poorly controlled type 1 diabetes. JTelemed Telecare 26:349-355

4. van den Boom L, Karges B, Auzanneau M, RamiMerhar B, Lilienthal E, von Sengbusch S, Datz N, Schroder C, Kapellen T, Laimer M et al (2019) Temporal Trends and Contemporary Use of Insulin Pump Therapy and Glucose Monitoring Among Children, Adolescents, and Adults With Type 1 Diabetes Between 1995 and 2017. Diabetes Care 42:2050-2056

5. Digitalisierungsreport 2021 - DUTReport. https:// www.dut-report.de/digitalisierungsreport-2021. Zugegriffen: 16.4.2021

6. Frielitz FS, Storm N, Hiort O, Katalinic A, von Sengbusch S (2019) The creation of a data protection policy: a guide to telemedicine healthcare projects. Bundesgesundheitsblatt Gesundheitsforschung Gesundheitsschutz 62:479-485

7. Bundesärztekammer (2021) Bundesärztekammer: Bekanntgaben der Herausgeber, Hinweise und Erläuterungen zu § 7 Abs.4 MBO-Ä - Behandlung im persönlichen Kontakt und Fernbehandlung. Dtsch Arztebl 116(19):A-978/B-810/C-798

8. Jorzig AS (2020) F: Videosprechstunde. In: Jorzig AS, F (Hrsg) Digitalisierung im Gesundheitswesen: Ein kompakter Streifzug durch Recht, Technikund Ethik. Springer, Berlin:, S185-191

9. Frielitz FSSS, Heinemann L, Freckmann G, von Sengbusch S (2020) Der Auftragsverarbeitungsvertrag (AV-Vertrag): Relevanz und praktische Bedeutung für die Diabetologie. Diabetol Stoffwechs 15:312-316

10. Bomba F, Muller-Godeffroy $E$, von Sengbusch $S$ (2018) Experiences in Sensor-Augmented Pump Therapy in Families with two Children with Type 1 diabetes: A Qualitative Study. Exp Clin Endocrinol Diabetes 126:162-167

11. Diabeter. https://diabeter.nl/. Zugegriffen: 16.4.2021

12. von Sengbusch $S$, Eisemann N, MuellerGodeffroy E, Lange K, Doerdelmann J, Er$\operatorname{dem}$ A, Menrath I, Bokelmann J, Krasmann M, Kaczmarczyk P et al (2020) Outcomes of monthly video consultations as an add-on to regular care for children with type 1 diabetes: A 6-month quasirandomized clinical trial followed by an extension phase. Pediatr Diabetes 21:1502-1515

13. Braune K, Boss K, Schmidt-Herzel J, Gajewska KA, Thieffry A, Schulze L, Posern B, Raile K (2021) Shaping Workflows in Digital and Remote Diabetes Care During the COVID-19 Pandemic: A Service Design Approach. JMIR Mhealth Uhealth 9(4):e24374. https://doi.org/10.2196/24374

14. Anderson RM, Funnell MM, Butler PM, Arnold MS, Fitzgerald JT, Feste CC (1995) Patient empower- 
ment. Results of a randomized controlled trial. Diabetes Care 18:943-949

15. Nordfeldt S, Angarne-Lindberg T, Nordwall M, Ekberg J, Bertero C (2013) As Facts and Chats Go Online, What Is Important for Adolescents with Type 1 Diabetes? Plos One 8:e67659

16. SpencerMS, KiefferEC, SincoB, PiattG, PalmisanoG, Hawkins J, Lebron A, Espitia N, Tang T, Funnell M, Heisler M (2018) Outcomes at 18 Months From a Community Health Worker and Peer Leader Diabetes Self-Management Program for Latino Adults. Diabetes Care 41:1414-1422

17. Kinderdiabeteslotse Schleswig-Holstein. http:// www.kinderdiabeteslotse-sh.de. Zugegriffen: 16.4.2021

\section{Die Bekämpfung von Diabetes und Adipositas muss vorrangiges Politikziel werden}

Jeder zweite Erwachsene in Deutschland ist übergewichtig, jeder vierte adipös. Weltweit hat sich in den letzten Jahrzehnten die Zahl der übergewichtigen Menschen erhöht, in Deutschland sogar verdreifacht. Grund dafür ist laut Professor Dr. med. Matthias Blüher, Vorstandsmitglied der DDG und Direktor des Helmholtz Instituts für Metabolismus-, Adipositas- und Gefäßforschung des Helmholtz Zentrums München an der Universitätsmedizin Leipzig, die hierzulande weit verbreitete ungesunde Ernährungsweise, der immobile Lebensstil und die hochtechnisierte Arbeitswelt, die Ernährung und Bewegung zusätzlich negativ beeinflusse.

Über 60 Krankheiten stehen im Zusammenhang mit Übergewicht - allen voran Diabetes, aber auch Krankheiten von Herz und Gefäßen, Leber oder Lunge, Beschwerden des Bewegungs-apparates sowie verschiedene Krebsarten. „Die Adipositas-Welle muss eingedämmt werden, sonst werden wir ganz abgesehen von den individuellen Schicksalen und Problemen auch volkswirtschaftliche Nachteile erleiden", warnt DDG Präsident Professor Dr. med. Andreas Neu. Denn Adipositas habe dramatische biologische und gesellschaftliche Folgen. Die Fehlzeiten wegen Arbeitsunfähigkeit, aber auch die Zahl der Frühverrentungen steigt bei Menschen mit starkem Übergewicht deutlich an. „30 Milliarden Euro Kosten entfallen jedes Jahr allein auf Gesundheitsmaßnahmen im Zusammenhang mit Adipositas", so Blüher. Die indirekten Kosten belaufen sich sogar auf 60 Milliarden jährlich.

Das jetzt geplante DMP Adipositas ist ein notwendiger Schritt, um die Versorgung der Menschen mit krankhaftem Übergewicht deutlich zu verbessern und damit auch die Zahl der Neuerkrankungen an Diabetes Typ 2 zu reduzieren. „Viele dachten lange Zeit, man müsse sich doch einfach mehr bewegen und weniger essen", sagt Blüher. Doch eine Krankheit lasse sich nicht mit Appellen bekämpfen. „Wir müssen verstehen, warum Menschen zu viel essen und sich zu wenig bewegen." Um krankmachende Verhaltensmuster aufzubrechen, brauche es ein multimodales Konzept aus Ernährung, Bewegung,
Verhaltenstherapie - gegebenenfalls auch einer medikamentösen und chirurgischen Therapie.

„Es ist wichtig, dass das künftige DMP Adipositas genau solche Konzepte bei der Versorgung von Menschen mit starkem Übergewicht unterstützt", so die Forderung der DDG. Daher sollten bei der Entwicklung des neuen Behandlungsprogramms etablierte Strukturen und Expertise aus dem DMP Diabetes Typ 2 genutzt werden. Damit bekämen Menschen mit Adipositas Zugang zu einer kontinuierlichen, strukturierten und qualitätsgesicherten Therapie. Vor allem die sektorenübergreifende und interdisziplinäre Verzahnung und das wirkungsvolle Zusammenspiel zwischen Ärzteschaft und Diabetes-Beratungsberufen bietet nicht nur optimale Voraussetzungen für die Begleitung von Menschen mit Diabetes, sondern auch mit Adipositas.

Das DMP Adipositas ist ein wichtiger Schritt zur Umsetzung der 2020 verabschiedeten Nationalen Diabetes Strategie. „Unverzichtbar sind aber auch verhältnispräventive Maßnahmen, die sicherstellen, dass Adipositas gar nicht erst auftritt", betont DDG Geschäftsführerin Barbara Bitzer: Es bedarf einer verbindlichen Lebensmittelkennzeichnung und eines Verbots von Werbung für ungesunde Lebensmittel, die sich an Kinder und Jugendliche richtet. Schon lange fordert die DDG auch die Einführung einer "Gesunden Mehrwertsteuer", die gesunde Lebensmittel mit geringem Anteil an Zucker, Fetten und/oder Salz steuerlich entlastet, eine Stunde Bewegung am Tag für Kinder und Jugendliche sowie verbindliche Ernährungsstandards für das Essen in Kitas und Schulen. Nur dadurch kann langfristig ein Durchbruch bei der Prävention von Adipositas und Diabetes erzielt werden. „Wie auch immer die künftige Bundesregierung zusammengesetzt sein wird: Ein konkreter Umsetzungsplan für die Bekämpfung dieser Krankheiten muss oberstes Politikziel werden", so Bitzer.

Quelle: www.deutsche-diabetesgesellschaft.de 